\title{
Differential Localization of Pain-Related and Pain-Unrelated Neural Responses for Acupuncture at BL60 Using BOLD fMRI
}

\author{
Na-Hee Kim, ${ }^{1}$ Seung-Yeon Cho, ${ }^{1}$ Geon-Ho Jahng, ${ }^{2}$ Chang-Woo Ryu, ${ }^{2}$ Seong-Uk Park, \\ Chang-Nam Ko, ${ }^{1}$ and Jung-Mi Park ${ }^{1,3}$ \\ ${ }^{1}$ Department of Cardiology and Neurology of Korean Medicine, College of Korean Medicine, Kyung Hee University, \\ Seoul, Republic of Korea \\ ${ }^{2}$ Department of Radiology, Kyung Hee University Hospital at Gangdong, School of Medicine, Kyung Hee University, \\ Seoul, Republic of Korea \\ ${ }^{3}$ Stroke and Neurological Disorders Center, Kyung Hee University Hospital at Gangdong, \\ 149 Sangil-dong, Gangdong-gu, Seoul 134-727, Republic of Korea \\ Correspondence should be addressed to Jung-Mi Park; pajama@khu.ac.kr
}

Received 12 April 2013; Accepted 31 May 2013

Academic Editor: David Baxter

Copyright (C) $2013 \mathrm{Na}$-Hee Kim et al. This is an open access article distributed under the Creative Commons Attribution License, which permits unrestricted use, distribution, and reproduction in any medium, provided the original work is properly cited.

\begin{abstract}
The objective of this study was to differentiate between pain-related and pain-unrelated neural responses of acupuncture at BL60 to investigate the specific effects of acupuncture. A total of 19 healthy volunteers were evaluated. fMRI was performed with sham or verum acupuncture stimulation at the left BL60 before and after local anesthesia. To investigate the relative BOLD signal effect for each session, a one-sample $t$-test was performed for individual contrast maps, and a paired $t$-test to investigate the differences between the pre- and post-anesthetic signal effects. Regarding verum acupuncture, areas that were more activated before local anesthesia included the superior, middle, and medial frontal gyri, inferior parietal lobule, superior temporal gyrus, thalamus, middle temporal gyrus, cingulate gyrus, culmen, and cerebellar tonsil. The postcentral gyrus was more deactivated before local anesthesia. After local anesthesia, the middle occipital gyrus, inferior temporal gyrus, postcentral gyrus, precuneus, superior parietal lobule, and declive were deactivated. Pre-anesthetic verum acupuncture at BL60 activated areas of vision and pain transmission. Postanesthetic verum acupuncture deactivated brain areas of visual function, which is considered to be a pain-unrelated acupuncture response. It indicates that specific effects of acupoint BL60 are to control vision sense as used in the clinical setting.
\end{abstract}

\section{Introduction}

Acupuncture is a widespread component of traditional medicine in Korea, China, and Japan and is characterized by stimulation via the insertion of needles, usually inducing some degree of pain. By noxious or external pressure sensation, the most commonly activated brain regions include the primary (S1) and secondary somatosensory (S2) cortices, as well as the prefrontal, anterior cingulate, temporal, and insular cortices $[1,2]$. These cortices of ascending somatosensory pathways of pain-related activation are due to the transmission of noxious stimuli along the spinohypothalamic pathway, the spinopontoamygdaloid pathway, and the spinothalamic pathway [3-5].

Functional magnetic resonance imaging (fMRI) has been used to indirectly detect brain functions $[6,7]$. Several human
fMRI studies have also shown that acupuncture needling affects brain activation. The relationship between acupoint and correspondent brain location has been discussed $[8,9]$, and different methods of stimulation such as tactile acupuncture or superficial pricking are associated with specific brain activation $[10,11]$. These results of neuronal activation suggest that acupuncture may be related to autonomic, cognitive, and affective functions, as well as sensorimotor control with connections to the cerebrum, brainstem, and other areas $[8,9,11-13]$.

Acupuncture-evoked neuronal stimulation still remains unclear because acupuncture is composed of various components including pain or pressure sensation, as well as placebo effect from anticipation, imagination, or concentration. In order to investigate the "specific" effect of either penetrating 
or nonpenetrating acupuncture, it is crucial to determine whether and to what extent the effect is related to a pain response.

Recently, fMRI studies have investigated acupoint at BL60 in a canine model [14]. Several fMRI studies using acupuncture at BL60 showed brain activation $[15,16]$, but, as mentioned earlier, the results did not distinguish whether the activated areas were related or unrelated to pain responses. To the best of our knowledge, no human studies have been published on fMRI activation in response to acupuncture before and after local anesthesia (i.e., with or without pain). Besides, there were some limitations from the previous canine model study [14]. We could not determine whether the dogs felt Deqi sensation and complete suppression of pain was uncertain.

Localization of pain-related and pain-unrelated responses to acupuncture is necessary in order to discover acupuncture's effect on pain sensation. The objective of this study was to determine the nature of pain related and pain unrelated in order to define specific neural responses to acupuncture using acupoint BL60.

\section{Materials and Methods}

2.1. Study Subjects. Twenty female subjects (mean age: $29.5 \pm$ 5.5 (SD) years; range: 21 to 43 years) consented to participate in this study. The inclusion criteria were as follows: (i) subjects had no past medical history or current history of physical or psychiatric diseases, (ii) they did not take and were currently not taking any prescribed or illicit drugs, and (iii) all subjected were right handed. They had experienced acupuncture. For each participant, fMRI was scheduled to avoid menstruation periods. Informed consent was obtained from all participants, and the protocol was approved by the Institutional Review Board (IRB) of Kyung Hee University Hospital at Gangdong (KHNMC-OH-IRB 2011-004). All experiments were conducted in accordance with the Declaration of Helsinki. One participant was excluded from the analysis because she was found to have multifocal T2 hyperintensive lesions in the subcortical white matter, which might be indicative of small vessel disease. The results of the remaining 19 participants were analyzed. None of the participants' head motion was greater than $5 \mathrm{~mm}$.

2.2. Definition and Manipulation of Acupoint BL60. The verum and sham acupuncture needling were applied at the same meridian BL60 acupoint (KonLyun in Korean, KunLun in Chinese) on the left [17]. It is located in the center of the depression between the prominence of the lateral malleolus of the fibula and the calcaneal tendon behind the ankle joint. For the verum acupuncture stimulation, a disposable stainless steel needle with a diameter of $0.25 \mathrm{~mm}$ and a length of $30 \mathrm{~mm}$ (DB106 Spring Handle, Dongbang Acupuncture Inc., Boryeong, Chungnam, Korea) was used. The verum acupuncture needle was inserted into the skin to a vertical depth of $1.0-1.5 \mathrm{~cm}$ in the deep tissue layer. For the sham acupuncture stimulation, a disposable placebo needle made of tungsten with a diameter of $0.25 \mathrm{~mm}$ and a length of $40 \mathrm{~mm}$
(Dongbang Acupuncture Inc., Boryeong, Chungnam, Korea) was used. The manipulation method consisted of constant tactile stimulation with a nonpenetrating needle to mimic acupuncture procedures. This procedure was performed by an experienced Korean medicine doctor and was conducted by the same person at each session.

2.3. Experimental Paradigms. Each study participant underwent four sessions in this study. The order of the four sessions included sham and verum acupuncture stimulations prior to receiving local anesthesia, followed by the same stimulations after receiving local anesthesia (sham-verumanesthesia-sham-verum). Figure 1 shows the block design of fMRI paradigm for stimulation. At baseline, a 30-second video clip of a leg was shown as visual stimulation (B). Visual stimulation then included showing a video clip of verum acupuncture treatment performed on the leg, which had been prepared beforehand (V). Each participant watched the video clip using a mirror inside the MRI machine and was asked to concentrate on the visual stimulation and not to sleep, move, or think of anything else. Sham or verum acupuncture stimulation of 30 seconds was performed at the BL60 acupoint in addition to showing the same video clip of a leg (S or A). The needle was manually rotated clockwise and counterclockwise at a frequency rate of twice per second $(2 \mathrm{~Hz})$. Thus, the block-designed paradigm for the sham subsession was B-V-S-B-V-S-B-V-S, and for the verum subsession was B-V-A-B-V-A-B-V-A.

After the first set of sham and verum sessions was completed, $0.2 \mathrm{~mL}$ of lidocaine $\mathrm{HCl}$ solution (Lidocaine $\mathrm{HCl}$ 2\%/20 mL, Huons, Jecheon, Republic Korea) was injected into the region of acupoint BL60 as a local anesthetic agent. After obtaining an anatomic image to confirm the spot, the effects of local anesthesia were tested by pricking and performing the second set of sham and verum sessions using the same techniques previously described. After the fMRI session was completed, participants were asked to recall and rate their pain intensity.

2.4. fMRI Acquisition. All fMRI images were acquired using a 3.0 Tesla whole body MR scanner (Acheiva, Philips Medical System, Best, The Netherlands) with an 8-channel head coil. Participants were placed into the magnet bore head first in a supine position, wearing helmets lined with soft foam padding to help reduce motion artifact.

To maximize the effect of BOLD, a gradient-echo planar imaging (EPI) sequence was used with the following parameters: imaging matrix size, $64 \times 64$ reconstructed to $96 \times$ 96 pixels; field of view $(\mathrm{FOV}), 230 \times 230 \mathrm{~mm}$; echo time (TE), $35 \mathrm{~ms}$; repetition time (TR), $3000 \mathrm{~ms}$; and flip angle, $90^{\circ}$; 25 slices $5 \mathrm{~mm}$ thick, without a gap between slices; voxel size, $3.6 \mathrm{~mm} \times 3.6 \mathrm{~mm} \times 5.0 \mathrm{~mm}$ reconstructed to $2.4 \mathrm{~mm} \times$ $2.4 \mathrm{~mm} \times 5.0 \mathrm{~mm}$, and total of 180 dynamic scans.

Furthermore, anatomical images were acquired with a 2-dimensional (2D) T2-weighted turbo spin echo sequence (TR, $3000 \mathrm{~ms}$; TE, $80 \mathrm{~ms}$; flip angle, 90 FOV, $230 \times 230 \mathrm{~mm}$; slice thickness, $3 \mathrm{~mm}$; matrix size, $256 \times 256$; voxel resolution, $0.9 \mathrm{~mm} \times 0.9 \mathrm{~mm} \times 3.00 \mathrm{~mm}$, and transverse orientation) 


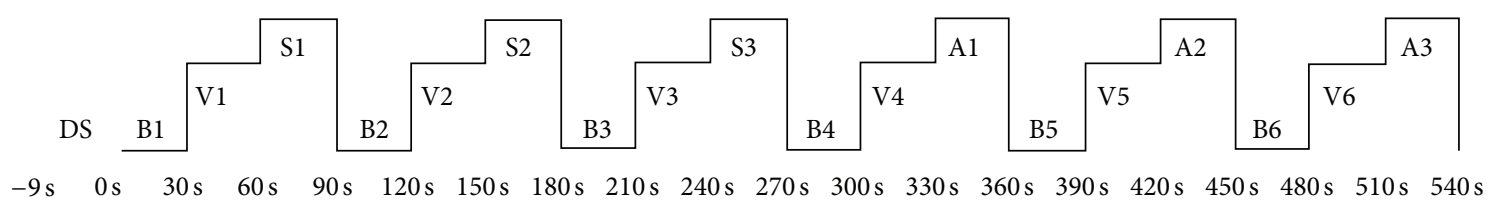

FIGURE 1: Block design of stimulations. A dummy scan was performed for 9 seconds before the experiment. Participants received alternating visual stimulations of the 30-second baseline video of the left leg (B) and the 30-second video of acupuncture treatment at the left BL60 (V). They then proceeded to undergo the 30-second sham acupuncture stimulation at BL60 with the same visual stimulation of the left leg (S) for 270 seconds followed by 30 seconds of $\mathrm{B}, 30$ seconds of $\mathrm{V}$, and 30 seconds of verum acupuncture at BL60 with the same visual stimulation of the left leg (A) for 270 seconds. DS: dummy scan, B: baseline stimulation, V: visual stimulation, S: sham acupuncture, A: verum acupuncture.

and a 3-dimensional (3D) T1-weighted (T1W) gradient echo sequence (TR, $9.9 \mathrm{~ms}$; TE, $4.6 \mathrm{~ms}$; flip angle, $8^{\circ}$; FOV, 240 $\times 240 \mathrm{~mm}$; slice thickness, $1 \mathrm{~mm}$; matrix size, $240 \times 240$; voxel resolution, $1.0 \mathrm{~mm} \times 1.0 \mathrm{~mm} \times 1.0 \mathrm{~mm}$, and sagittal orientation). The anatomical scan was performed shortly after local anesthesia using a lidocaine $\mathrm{HCl}$ injection into BL60. The total time for $2 \mathrm{D}$ and $3 \mathrm{D}$ anatomical imaging was 9 minutes and 47 seconds.

2.5. Processing of $f M R I$ Data. fMRI data analysis was performed using Statistical Parametric Mapping software (SPM5; Wellcome Department of Cognitive Neurology, London, UK; http://www.fil.ion.ucl.ac.uk/spm/) by way of MATLAB (Math Works Inc., Natick, MA). Head motion during the fMRI acquisition was calculated by rotation and translation on $x, y$, and $z$ coordinates and realigned automatically using three-dimensional motion correction to minimize the movement-related variance of the subject. Subjects whose heads moved excessively $(>5 \mathrm{~mm}$ ) were excluded from the analysis. Realigned anatomical MR images and fMRI images for each participant were coregistered to the 3D-T1W image. The 3D-T1W image and fMRI data were spatially normalized into the SPM Montreal Neurological Institute (MNI) template. Anatomical 3D MR images were standardized using standard anatomical space devised by Talairach and Tournoux, and fMRI images were standardized with the same process as the anatomical images. The spatially normalized image volumes were smoothed using a Gaussian kernel of the full width at half maximum (FWHM) of 8 $\times 8 \times 10 \mathrm{~mm}$ to raise the power of discrimination among noises of MR signal changes classified by voxels produced by hemodynamic response and to set match statistical model offered by SPM.

2.6. Statistical Analysis of fMRI Data. For the individual level analysis, in order to obtain activated or deactivated contrast maps, the stimulation conditions were compared to the baseline conditions for each session and each subject using a general linear model. For the sham subsession, the following contrast maps were obtained:

(i) sham acupuncture (S) > baseline (B), which were greater during the sham acupuncture stimulations than during the baseline visual stimulation before local anesthesia,

(ii) sham acupuncture (S) < baseline (B), contrariwise.
For the verum acupuncture subsession, the following contrast maps were obtained:

(iii) acupuncture (A) > baseline (B) for activation maps,

(iv) acupuncture (A) < baseline (B) for deactivation maps.

The contrast maps after local anesthesia were also obtained in the same way.

For the group level analysis, the activation or deactivation contrast maps obtained from the individual level analysis were used. In order to investigate the BOLD signal effect for each session, a one-sample $t$-test was performed for the 8 different contrast maps with a statistical threshold of $P=0.001$, without multiple compensations, and with a cluster size $\geq 10$ voxels. The result areas were limited to the grey matter with an explicit mask of grey matter (expression threshold: IL > 0.5).

To investigate the pain-related effects and pain-unrelated effects, the differences between the pre- and post-anesthetic BOLD signal effects were also compared using a paired $t$ test. To investigate the acupuncture-specific or sham-specific effects, the sham and verum acupuncture stimulation blocks in each pre- and post-anesthetic session were included.

2.7. Questionnaire of Self-Reported Sensations. Self-reported sensation was documented immediately after the MRI acquisition using a 0 to 10 numeric rating scale (NRS). To verify the successfulness of blinding, a self-reported guess of what happened during the visual stimulation of acupuncture (V) when no acupuncture was actually performed was also used.

\section{Results}

3.1. Areas of Activation and Deactivation of Each Session. The differences in BOLD signals between the sham acupuncture block (S) and the baseline stimulation block (B) and between the verum acupuncture block (A) and the baseline stimulation block (B) were investigated; this was considered the additional change from the baseline. Table 1 shows different activated/deactivated areas between the pre- and postanesthetic sham acupuncture. Table 2 shows different activated/deactivated areas between the pre- and post-anesthetic verum acupuncture (Figure 2).

3.2. Results of Self-Reported Sensations. Table 3 shows the type of sensation and mean (standard deviation) of each NRS score reported by study participants. 
TABLE 1: Activated and deactivated brain areas during the sham acupuncture before and after anesthesia.

\begin{tabular}{|c|c|c|c|c|c|}
\hline & Area $(x, y, z)$ & Side & $\mathrm{KE}$ & $T$ peak & $Z$ score \\
\hline \multirow{11}{*}{$\begin{array}{l}\text { Sham }>\text { base } \\
\text { (pre-anesthetic) }\end{array}$} & Cortex & & & & \\
\hline & Frontal lobe, inferior frontal gyrus $(-50,8,16)$ & $\mathrm{L}$ & 213 & 6.81 & 4.73 \\
\hline & Frontal lobe, inferior frontal gyrus $(40,20,8)$ & $\mathrm{R}$ & 685 & 7.49 & 4.98 \\
\hline & $\begin{array}{l}\text { Temporal lobe, superior temporal gyrus } \\
\text { BA } 22(-60,-58,16)\end{array}$ & $\mathrm{L}$ & 2341 & 10.87 & 5.97 \\
\hline & $\begin{array}{l}\text { Temporal lobe, superior temporal gyrus } \\
\text { BA } 22(66,-44,8)\end{array}$ & $\mathrm{R}$ & 3103 & 8.64 & 5.57 \\
\hline & $\begin{array}{l}\text { Parietal lobe, inferior parietal lobule } \\
(-60,-32,34)\end{array}$ & $\mathrm{L}$ & 2341 & 7.05 & 4.82 \\
\hline & $\begin{array}{l}\text { Limbic lobe, cingulate gyrus } \\
\text { BA } 24(-4,12,28)\end{array}$ & $\mathrm{L}$ & 10039 & 9.13 & 5.51 \\
\hline & Sublobar, claustrum $(-34,0,-4)$ & $\mathrm{L}$ & 793 & 8.34 & 5.27 \\
\hline & Sublobar, caudate $(-14,10,6)$ & $\mathrm{L}$ & 533 & 6.91 & 4.77 \\
\hline & Cerebellum & & & & \\
\hline & Anterior lobe, culmen $(-2,-60,4)$ & $\mathrm{L}$ & 10039 & 9.07 & 5.49 \\
\hline \multirow{5}{*}{$\begin{array}{l}\text { Sham }>\text { base } \\
\text { (post-anesthetic) }\end{array}$} & Cortex & & & & \\
\hline & Occipital lobe, precuneus $(-14,-64,28)$ & $\mathrm{L}$ & 54 & 4.63 & 3.71 \\
\hline & $\begin{array}{l}\text { Temporal lobe, middle temporal gyrus } \\
(-46,2,-18)\end{array}$ & $\mathrm{L}$ & 10 & 4.61 & 3.70 \\
\hline & Cerebellum & & & & \\
\hline & Anterior lobe, culmen $(0,-56,-8)$ & $\mathrm{L}$ & 37 & 4.05 & 3.37 \\
\hline \multirow{3}{*}{$\begin{array}{l}\text { Sham }<\text { base } \\
\text { (pre-anesthetic) }\end{array}$} & Cortex & & & & \\
\hline & Frontal lobe, precentral gyrus $(-44,-14,56)$ & $\mathrm{L}$ & 37 & 4.19 & 3.46 \\
\hline & $\begin{array}{l}\text { Parietal lobe, postcentral gyrus } \\
\text { BA } 3(-38,-22,48)\end{array}$ & $\mathrm{L}$ & 37 & 4.19 & 3.46 \\
\hline \multirow{5}{*}{$\begin{array}{l}\text { Sham }<\text { base } \\
\text { (post-anesthetic) }\end{array}$} & Cortex & & & & \\
\hline & Occipital lobe, middle occipital gyrus & $\mathrm{L}$ & & & \\
\hline & $(-24,-88,20)$ & & 59 & 4.77 & 3.79 \\
\hline & $(-48,-74,2)$ & & 63 & 4.46 & 3.62 \\
\hline & $\begin{array}{l}\text { Frontal lobe, precentral gyrus } \\
\text { BA } 6(-40,-12,58)\end{array}$ & $\mathrm{L}$ & 27 & 4.25 & 3.49 \\
\hline
\end{tabular}

$P_{\text {uncorrected }}($ cluster level $)<0.001$, cluster size $\geq 10$ voxels.

Sham: sham acupuncture; base: baseline visual stimulation by showing a video clip of a leg; R: right; L: left; KE: expected voxels per cluster.

\section{Discussion}

In this study, differences between pain-related and painunrelated brain activation during acupuncture stimulation at the left BL60 were investigated. Pre-anesthetic brain activity included pain response, and post-anesthetic brain activity was related to pain-unrelated responses. To verify neuronalspecific effects of acupuncture, the differences between acupuncture sessions and baseline sessions (visual stimulation) were assessed. Therefore, the activated/deactivated brain areas discussed were presumed to be "acupuncturespecific effects only," which are distinct from accessory effects such as anticipation, imagination, emotion, concentration, interaction with the doctor, and stimulation from the supine position. These accessory factors have been argued in previous studies $[15,18]$.
Verum or sham acupuncture at BL60 activated some regions in the frontal, temporal, parietal, and limbic lobes of the cortex and cerebellum before local anesthesia. It is thought that sensory inputs by acupuncture stimulation are transferred to the frontal cortex through long-range cortical tracts, and the regions in the frontal cortex send and receive signals with the cingulate gyrus and thalamus.

In activated areas associated with pre-anesthetic verum or sham acupuncture stimulations, the inferior parietal lobule (IPL) and superior temporal gyrus (STG) have been involved in the perception of emotions in facial stimuli and the interpretation of sensory information [19, 20]. More specifically, the superior frontal gyrus is involved in selfawareness and coordinating actions with the sensory system [21]. The middle frontal gyrus, especially Brodmann area (BA) 8 including the frontal eye fields, is believed to play 
TABLE 2: Activated and deactivated brain areas during the verum acupuncture before and after anesthesia.

\begin{tabular}{|c|c|c|c|c|c|}
\hline & Area $(x, y, z)$ & Side & $\mathrm{KE}$ & $T$ peak & $Z$ score \\
\hline \multirow{17}{*}{$\begin{array}{l}\text { Acup }>\text { base } \\
\text { (pre-anesthetic) }\end{array}$} & Cortex & & & & \\
\hline & Frontal lobe, superior frontal gyrus & $\mathrm{L}$ & & & \\
\hline & BA $8(-16,34,48)$ & & 24 & 5.32 & 4.07 \\
\hline & $(0,54,26)$ & & 12 & 3.82 & 3.23 \\
\hline & $\begin{array}{l}\text { Frontal lobe, middle frontal gyrus } \\
\text { BA } 8(-40,16,44)\end{array}$ & $\mathrm{L}$ & 33 & 5.80 & 4.30 \\
\hline & $\begin{array}{l}\text { Frontal lobe, medial frontal gyrus } \\
(2,42,22)\end{array}$ & $\mathrm{R}$ & 14 & 3.77 & 3.20 \\
\hline & $\begin{array}{l}\text { Parietal lobe, inferior parietal lobule } \\
\text { BA } 40(-62,-34,30)\end{array}$ & $\mathrm{L}$ & 127 & 5.65 & 4.23 \\
\hline & $\begin{array}{l}\text { Limbic lobe, posterior cingulate } \\
\text { BA } 40(0,-62,8)\end{array}$ & $\mathrm{L}$ & 177 & 5.11 & 3.97 \\
\hline & $\begin{array}{l}\text { Temporal lobe, superior temporal gyrus } \\
\text { BA } 22(64,-48,20)\end{array}$ & $\mathrm{R}$ & 54 & 4.61 & 3.70 \\
\hline & Sub-lobar, thalamus $(8,-10,-2)$ & $\mathrm{R}$ & 17 & 4.41 & 3.59 \\
\hline & Temporal lobe, middle temporal gyrus & $\mathrm{R}$ & & & \\
\hline & $(54,-24,-8)$ & & 14 & 4.33 & 3.54 \\
\hline & $(56,-42,-2)$ & & 31 & 3.97 & 3.32 \\
\hline & $\begin{array}{l}\text { Limbic lobe, cingulate gyrus } \\
\text { BA } 24(0,-22,36)\end{array}$ & $\mathrm{L}$ & 11 & 3.88 & 3.27 \\
\hline & Cerebellum & & & & \\
\hline & Anterior lobe, culmen $(0,-52,-4)$ & $\mathrm{L}$ & 35 & 4.64 & 3.72 \\
\hline & Posterior lobe, cerebellar tonsil $(-2,-58,-38)$ & $\mathrm{L}$ & 24 & 4.11 & 3.41 \\
\hline $\begin{array}{l}\text { Acup }>\text { base } \\
\text { (post-anesthetic) }\end{array}$ & None & & & & \\
\hline \multirow{2}{*}{$\begin{array}{l}\text { Acup }<\text { base } \\
\text { (pre-anesthetic) }\end{array}$} & Cortex & & & & \\
\hline & $\begin{array}{l}\text { Parietal lobe, postcentral gyrus } \\
\text { BA } 1(-52,-18,50)\end{array}$ & $\mathrm{L}$ & 14 & 4.02 & 3.35 \\
\hline \multirow{9}{*}{$\begin{array}{l}\text { Acup < base } \\
\text { (post-anesthetic) }\end{array}$} & Cortex & & & & \\
\hline & $\begin{array}{l}\text { Occipital lobe, middle occipital gyrus } \\
(46,-72,-8)\end{array}$ & $\mathrm{R}$ & 1144 & 6.11 & 4.44 \\
\hline & $\begin{array}{l}\text { Occipital lobe, middle occipital gyrus } \\
(-24,-88,20)\end{array}$ & $\mathrm{L}$ & 59 & 4.77 & 3.39 \\
\hline & $\begin{array}{l}\text { Occipital lobe, inferior temporal gyrus } \\
(-48,-72,2)\end{array}$ & $\mathrm{L}$ & 63 & 4.46 & 3.62 \\
\hline & $\begin{array}{l}\text { Parietal lobe, postcentral gyrus } \\
\text { BA } 40(40,-32,54)\end{array}$ & $\mathrm{R}$ & 83 & 4.79 & 3.80 \\
\hline & $\begin{array}{l}\text { Parietal lobe, precuneus } \\
\text { BA } 7(-12,-40,52)\end{array}$ & $\mathrm{L}$ & 47 & 4.27 & 3.50 \\
\hline & $\begin{array}{l}\text { Parietal lobe, superior parietal lobule } \\
\text { BA } 7(-22,-66,46)\end{array}$ & $\mathrm{L}$ & 10 & 3.95 & 3.31 \\
\hline & Cerebellum & & & & \\
\hline & Posterior lobe, declive $(-30,-64,-14)$ & $\mathrm{L}$ & 427 & 6.60 & 4.65 \\
\hline
\end{tabular}

$P_{\text {uncorrected }}$ (cluster level) $<0.001$, cluster size $\geq 10$ voxels.

Acup: verum acupuncture; base: baseline visual stimulation by showing a video clip of a leg; R: right; L: left; KE: expected voxels per cluster.

an important role in the control of eye movements. BL60 acupoint has been shown to be involved in visual information processing [22].

Moreover, pre-anesthetic verum acupuncture involved activation of the medial frontal gyrus associated with high- level executive functions and decision-related processes [23], as well as the tonsil or culmen, where ipsilateral eye movement is controlled unconsciously.

Brain areas that were more activated in pre-anesthetic verum than in sham procedures included the cingulate gyrus 


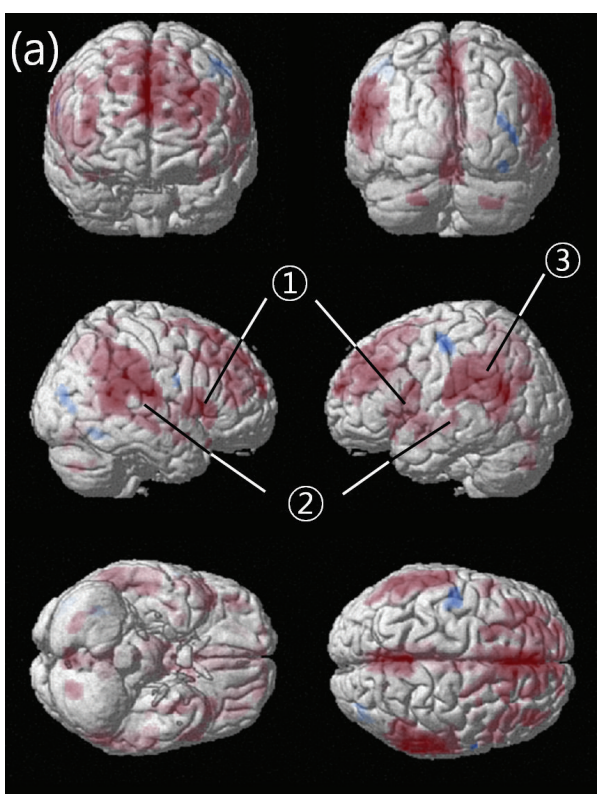

(a)

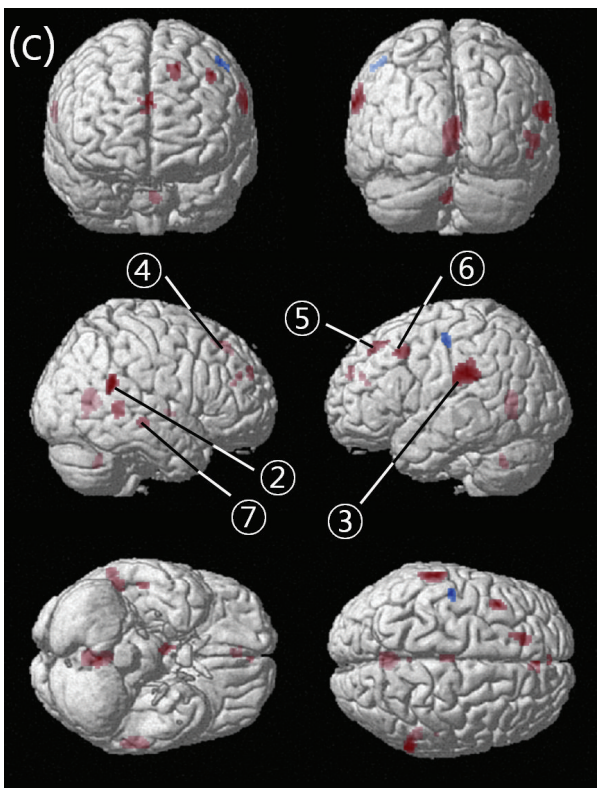

(c)

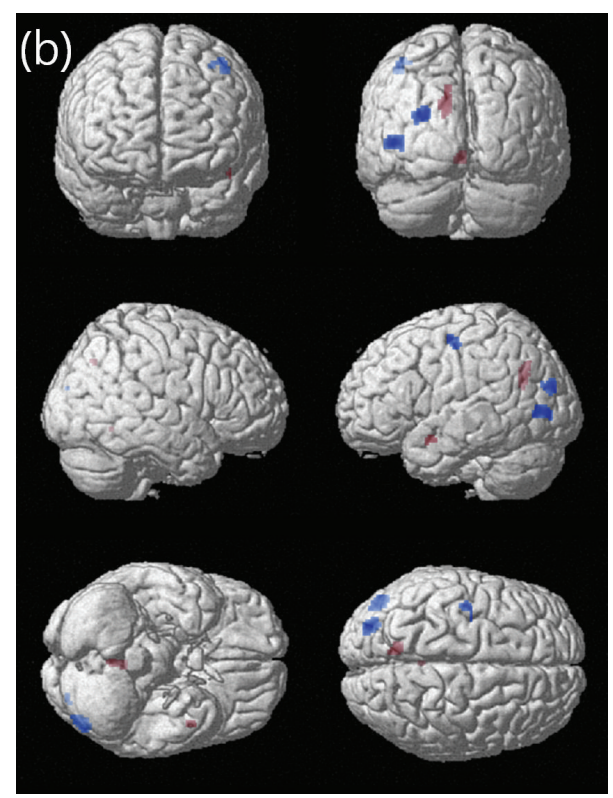

(b)

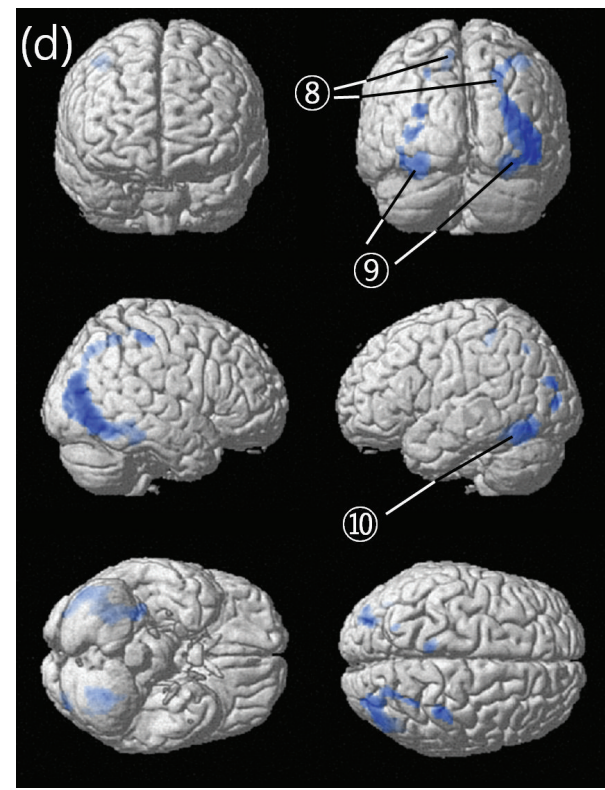

(d)

FIGURE 2: Brain activity during the sham or verum sessions before and after anesthesia. (a) Activated and deactivated brain areas during the sham session before anesthesia. (b) Activated and deactivated brain areas during the sham session after anesthesia. (c) Activated and deactivated brain areas during the verum session before anesthesia. (d) Activated and deactivated brain areas during the verum session after anesthesia. (1) Inferior frontal gyrus (R, L), (2) superior temporal gyrus (R, L), (3) inferior parietal lobule (L), (4) medial frontal gyrus (R), (5) superior frontal gyrus (L), (6) middle frontal gyrus (L), (7) middle temporal gyrus (R), (8) postcentral gyrus (L, R), (9) middle occipital gyrus (L, R), (10) inferior temporal gyrus (L). R: right, L: left. Activated areas are expressed in red, and deactivated areas are expressed in blue. Baseline stimulation is showing a video clip of a leg.

and thalamus, suggesting an association with the known pain transmission pathway [24] or the unpleasantness transmission pathway [25].

On the other hand, the postcentral gyrus was deactivated by verum or sham acupuncture before anesthesia. It is located in the primary somatosensory cortex (S1), the main sensory receptive area for the sense of touch, and receives thalamocortical projections from sensory input fields. The deactivation area in the talairach coordinate $(x=-38, y=$ $-22, z=48$ and $x=-52, y=-18, z=50)$ is where the S1 somatotopic eye representation may exist [26] indicating that acupuncture at BL60 may control eye sensations, in 
TABLE 3: Self-reported sensations using a 0 to 10 numeric rating scale (NRS) and the mean (standard deviation) of each NRS score.

\begin{tabular}{|c|c|c|c|c|}
\hline \multirow{2}{*}{ Type of sensation } & \multicolumn{2}{|c|}{ Mean (standard deviation) } & \multirow{2}{*}{ Differences of average rating (Verum-Sham) ${ }^{*}$} & \multirow{2}{*}{$P$-value } \\
\hline & Sham acupuncture & Verum acupuncture & & \\
\hline \multicolumn{5}{|c|}{$\begin{array}{l}\text { The moment when the needle } \\
\text { touches the skin }\end{array}$} \\
\hline Hurting & $1.15(2.39)$ & $2.95(2.74)$ & 1.80 & $0.002^{*}$ \\
\hline Penetrating & $3.40(2.64)$ & $5.65(2.50)$ & 2.25 & $0.002^{*}$ \\
\hline Sharp pain & $2.65(1.83)$ & $4.90(2.85)$ & 2.25 & $<0.001^{*}$ \\
\hline Aching & $1.75(2.02)$ & $3.95(2.74)$ & 2.20 & $<0.001^{*}$ \\
\hline Subtotal average & 2.24 & 4.36 & 2.12 & $<0.001^{*}$ \\
\hline \multicolumn{5}{|c|}{$\begin{array}{l}\text { Throughout the acupuncture } \\
\text { stimulation }\end{array}$} \\
\hline Aching & $2.10(2.18)$ & $3.80(2.07)$ & 1.70 & $<0.001^{*}$ \\
\hline Soreness & $1.65(1.95)$ & $2.80(2.78)$ & 1.15 & $0.013^{*}$ \\
\hline Deep pressure & $1.70(2.20)$ & $3.95(2.61)$ & 2.25 & $<0.001^{*}$ \\
\hline Heaviness & $2.25(1.70)$ & $3.85(2.68)$ & 1.60 & $0.004^{*}$ \\
\hline Fullness (distention) & $1.45(2.48)$ & $3.50(2.46)$ & 2.05 & $<0.001^{*}$ \\
\hline Tingling & $2.35(1.34)$ & $4.25(3.35)$ & 1.90 & $0.014^{*}$ \\
\hline Warmth & $0.70(1.80)$ & $1.15(2.21)$ & 0.45 & 0.119 \\
\hline Cold & $1.25(2.26)$ & $1.65(2.32)$ & 0.40 & 0.258 \\
\hline Numbness & $2.20(1.84)$ & $2.40(3.02)$ & 0.20 & 0.725 \\
\hline Dull pain & $2.00(1.64)$ & $2.80(2.82)$ & 0.80 & 0.084 \\
\hline Throbbing & $1.05(2.14)$ & $2.10(2.63)$ & 1.05 & $0.011^{*}$ \\
\hline Sharp pain & $2.20(2.13)$ & $4.35(3.41)$ & 2.15 & $0.005^{*}$ \\
\hline Spread & $2.10(2.88)$ & $3.25(2.83)$ & 1.15 & $0.034^{*}$ \\
\hline Anxiety & $2.75(1.38)$ & $4.75(3.39)$ & 2.00 & $0.002^{*}$ \\
\hline Other sensation & $0.70(1.52)$ & $1.40(2.39)$ & 0.70 & 0.090 \\
\hline Subtotal average & 1.76 & 3.07 & 1.31 & $<0.001^{*}$ \\
\hline Total average & 1.86 & 3.34 & 1.48 & $<0.001^{*}$ \\
\hline
\end{tabular}

For each sensation, a paired $t$-test was performed to compare the score between the sham and the verum acupuncture stimulation.

${ }^{*} P<0.05$.

addition to the sense of vision. The results above show the effects of acupuncture at BL60 including pain response. However, involvement of other areas might imply that verum acupuncture is associated with higher coordination function including vision.

Post-anesthetic verum acupuncture, which is considered to be a pain-unrelated mechanism, showed deactivation of the middle occipital gyrus, inferior temporal gyrus, precuneus, and superior parietal lobule. The occipital lobe is the visual processing center containing most of the anatomical region of the visual cortex. The inferior temporal gyrus, which is connected behind the inferior occipital gyrus, is one of the higher levels of the ventral stream of visual processing and may also be involved in face perception. The precuneus is also involved in visuospatial processing as well as episodic memory, and the superior parietal lobule is involved with spatial orientation and receives a great deal of visual input as well as sensory input from one's hand.

Therefore, these results confirm the specificity of acupoint BL60 as suggested in previous studies and suggest the clinical usefulness of BL60 in body reorientation via a pain-related or pain-unrelated pathway.

Self-reported sensation was stronger in verum acupuncture than in sham procedures in all categories, whereas brain activation appeared to be broader in sham acupuncture than in verum acupuncture. Even though needle insertion is more painful and induces more intense sensations, continuous tactile stimulation appears to activate a broader brain area. According to a previous fMRI study with acupuncture and superficial pricking, multiple distributed activation was noted in superficial pricking [13] (similar to the sham acupuncture in the present study). Our results suggest that broader brain activation of sham acupuncture may be associated with repetitive superficial pricking compared to verum acupuncture.

Previous studies reported that acupuncture manipulation was associated with decreased fMRI signals [11, 12]. Deactivation in such instances may be interpreted as the suppression of neuronal activity [27] due to the hemodynamic or metabolic effects of downregulation of accompanying 
neuronal inhibition [8]. The deactivation of limbic-cerebellar areas can be one of the effects of acupuncture [24]. The results of the present study are in agreement with previous fMRI studies of "acupuncture deactivation."

Traditionally, the acupoint BL60 has been used for disorders of upper or lower extremity, low back, arthritis of the hock, soft tissue injuries, and for eye-related disorders such as visual dizziness, redness, swelling of the eyes, as well as bursting eye pain $[6,18]$. Recent research showed that this acupoint activates bilateral regions within the visual cortex through interhemispheric visual-visual transmission, as well as adjacent regions in nonvisual cortices [16]. Additionally, tempospatial analysis showed vision-related acupoint specificity, including BL60 [15].

According to the present study, needling BL60 appeared to activate and deactivate various brain areas and was especially related to more vision control, as well as being involved in higher-order perception and fine regulation of emotion and information. The pain-unrelated response included deactivation of the middle occipital gyrus, postcentral gyrus, precuneus, superior parietal lobule, and cerebellar declive. The pain-related response included activation of the thalamus and cingulate gyrus, as well as superior and medial frontal gyri and middle temporal gyrus. Additionally, the left cerebellar culmen was commonly activated in the pre-anesthetic sham and verum acupunctures and post-anesthetic sham acupuncture, which might suggest a specific response to acupuncture with or without pain.

\section{Conclusions}

Pre-anesthetic verum acupuncture at BL60 activated areas of the brain associated with vision, as well as pain transmission; these findings are tantamount to a pain-related acupuncture response. Post-anesthetic verum acupuncture at BL60 deactivated brain areas of visual function, which is considered to be a pain-unrelated acupuncture response. These results indicate that specific effects of acupoint BL60 are to control visual sense as used in the clinical setting.

\section{Conflict of Interests}

No competing financial interests exist.

\section{Acknowledgment}

This work was supported by a Grant from Kyung Hee University in 2011 (KHU-20110919).

\section{References}

[1] C. Creac'h, P. Henry, J. M. Caille, and M. Allard, "Functional MR imaging analysis of pain-related brain activation after acute mechanical stimulation," American Journal of Neuroradiology, vol. 21, no. 8, pp. 1402-1406, 2000.

[2] A. K. P. Jones, W. D. Brown, K. J. Friston, L. Y. Qi, and R. S. J. Frackowiak, "Cortical and subcortical localization of response to pain in man using positron emission tomography,"
Proceedings of the Royal Society B, vol. 244, no. 1309, pp. 39-44, 1991.

[3] J. F. Bernard and J. M. Besson, "The spino(trigemino)pontoamygdaloid pathway: electrophysiological evidence for an involvement in pain processes," Journal of Neurophysiology, vol. 63, no. 3, pp. 473-490, 1990.

[4] R. Burstein, K. D. Cliffer, and G. J. Giesler Jr., "Direct somatosensory projections from the spinal cord to the hypothalamus and telencephalon," Journal of Neuroscience, vol. 7, no. 12, pp. 4159-4164, 1987.

[5] R. K. Fulbright, C. J. Troche, P. Skudlarski, J. C. Gore, and B. E. Wexler, "Functional MR imaging of regional brain activation associated with the affective experience of pain," American Journal of Roentgenology, vol. 177, no. 5, pp. 1205-1210, 2001.

[6] S.-U. Park, A.-S. Shin, G.-H. Jahng, S.-K. Moon, and J.-M. Park, "Effects of scalp acupuncture versus upper and lower limb acupuncture on signal activation of blood oxygen level dependent (BOLD) fMRI of the brain and somatosensory cortex," Journal of Alternative and Complementary Medicine, vol. 15, no. 11, pp. 1193-1200, 2009.

[7] T. Yoshiura, O. Wu, and A. G. Sorensen, "Advanced MR techniques. Diffusion MR imaging, perfusion MR imaging, and spectroscopy," Neuroimaging Clinics of North America, vol. 9, no. 3, pp. 439-453, 1999.

[8] K. K. S. Hui, J. Liu, O. Marina et al., "The integrated response of the human cerebro-cerebellar and limbic systems to acupuncture stimulation at ST 36 as evidenced by fMRI," NeuroImage, vol. 27, no. 3, pp. 479-496, 2005.

[9] S.-S. Jeun, J.-S. Kim, B.-S. Kim et al., "Acupuncture stimulation for motor cortex activities: a 3T fMRI Study," American Journal of Chinese Medicine, vol. 33, no. 4, pp. 573-578, 2005.

[10] S.-Y. Cho, G.-H. Jahng, S.-U. Park, W.-S. Jung, S.-K. Moon, and J.-M. Park, "FMRI study of effect on brain activity according to stimulation method at LI11, ST36: painful pressure and acupuncture stimulation of same acupoints," Journal of Alternative and Complementary Medicine, vol. 16, no. 4, pp. 489-495, 2010.

[11] S.-S. Yoo, E.-K. Teh, R. A. Blinder, and F. A. Jolesz, "Modulation of cerebellar activities by acupuncture stimulation: evidence from fMRI study," NeuroImage, vol. 22, no. 2, pp. 932-940, 2004.

[12] K. K. Hui, J. Liu, N. Makris et al., "Acupuncture modulates the limbic system and subcortical gray structures of the human brain: evidence from fMRI studies in normal subjects," Human Brain Mapping, vol. 9, no. 1, pp. 13-25, 2000.

[13] M.-T. Wu, J.-C. Hsieh, J. Xiong et al., "Central nervous pathway for acupunture stimulation: localization of processing with functional MR imaging of the brain-preliminary experience," Radiology, vol. 212, no. 1, pp. 133-141, 1999.

[14] S. K. Chang, G. H. Jahng, S. H. Lee, I. W. Choi, C. B. Choi, and W. S. Choi, "Differential localization of pain-related neural responses during acupuncture stimulation using Blood Oxygen Level Dependent (BOLD) fMRI in a canine model," The American Journal of Chinese Medicine, vol. 40, no. 5, pp. 919936, 2012.

[15] M. Dong, W. Qin, J. Sun et al., “Tempo-spatial analysis of visionrelated acupoint specificity in the occipital lobe using fMRI: an ICA study," Brain Research, vol. 1436, pp. 34-42, 2012.

[16] G. Li, R. T. F. Cheung, Q.-Y. Ma, and E. S. Yang, "Visual cortical activations on fMRI upon stimulation of the vision-implicated acupoints," NeuroReport, vol. 14, no. 5, pp. 669-673, 2003. 
[17] WHO Regional Office for the Western Pacific, "WHO standard acupuncture point locations in the Western Pacific Region," World Health Organization.

[18] N. Shankar, A. Varshney, A. Bhattacharya, and K. N. Sharma, "Electroacupuncture, morphine and clonidine: a comparative study of analgesic effects," Indian Journal of Physiology and Pharmacology, vol. 40, no. 3, pp. 225-230, 1996.

[19] E. D. Bigler, S. Mortensen, E. S. Neeley et al., "Superior temporal gyrus, language function, and autism," Developmental Neuropsychology, vol. 31, no. 2, pp. 217-238, 2007.

[20] J. Radua, M. L. Phillips, T. Russell et al., "Neural response to specific components of fearful faces in healthy and schizophrenic adults," NeuroImage, vol. 49, no. 1, pp. 939-946, 2010.

[21] I. I. Goldberg, M. Harel, and R. Malach, "When the brain loses its self: prefrontal inactivation during sensorimotor processing," Neuron, vol. 50, no. 2, pp. 329-339, 2006.

[22] K. H. Chang, R. Won, I. Shim, H. Lee, and B. H. Lee, "Effects of electroacupuncture at BL60 on formalin-induced Pain in Rats," Evidence-Based Complementary and Alternative Medicine, vol. 2012, Article ID 324039, 7 pages, 2012.

[23] A. Talati and J. Hirsch, "Functional specialization within the medial frontal gyrus for perceptual go/no-go decisions based on "what," "when," and "where" related information: an fMRI study," Journal of Cognitive Neuroscience, vol. 17, no. 7, pp. 981993, 2005.

[24] T. D. Wager, J. K. Rilling, E. E. Smith et al., "Placebo-induced changes in $\mathrm{FMRI}$ in the anticipation and experience of Pain," Science, vol. 303, no. 5661, pp. 1162-1167, 2004.

[25] F. Benuzzi, F. Lui, D. Duzzi, P. F. Nichelli, and C. A. Porro, "Does it look painful or disgusting? Ask your parietal and cingulate cortex," The Journal of Neuroscience, vol. 28, no. 4, pp. 923-931, 2008.

[26] D. van Westen, P. Fransson, J. Olsrud, B. Rosén, G. Lundborg, and E.-M. Larsson, "Fingersomatotopy in area 3b: an fMRIstudy," BMC Neuroscience, vol. 5, article 28, 2004.

[27] P. Fransson, G. Krüger, K.-D. Merboldt, and J. Frahm, "MRI of functional deactivation: temporal and spatial characteristics of oxygenation-sensitive responses in human visual cortex," NeuroImage, vol. 9, no. 6 I, pp. 611-618, 1999. 


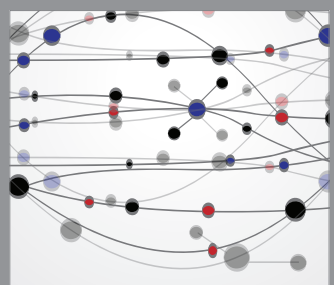

The Scientific World Journal
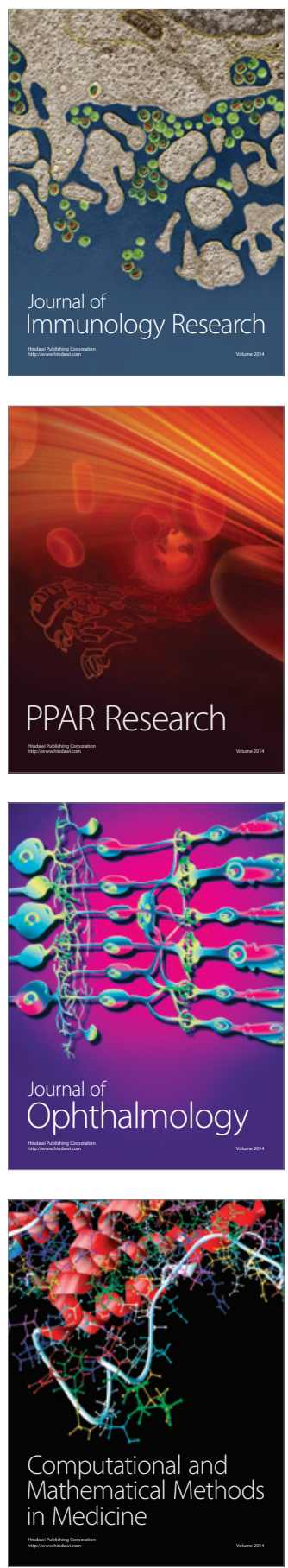

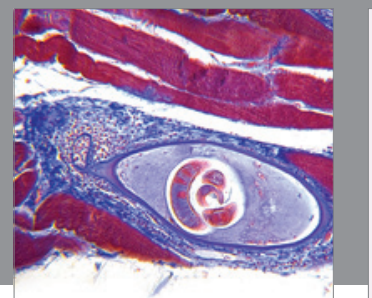

Gastroenterology

Research and Practice
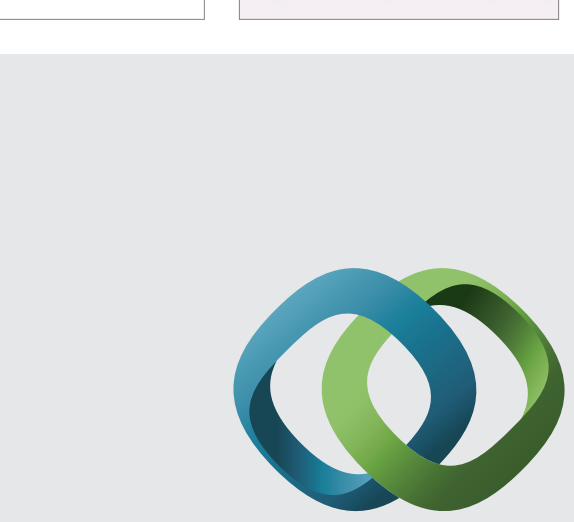

\section{Hindawi}

Submit your manuscripts at

http://www.hindawi.com
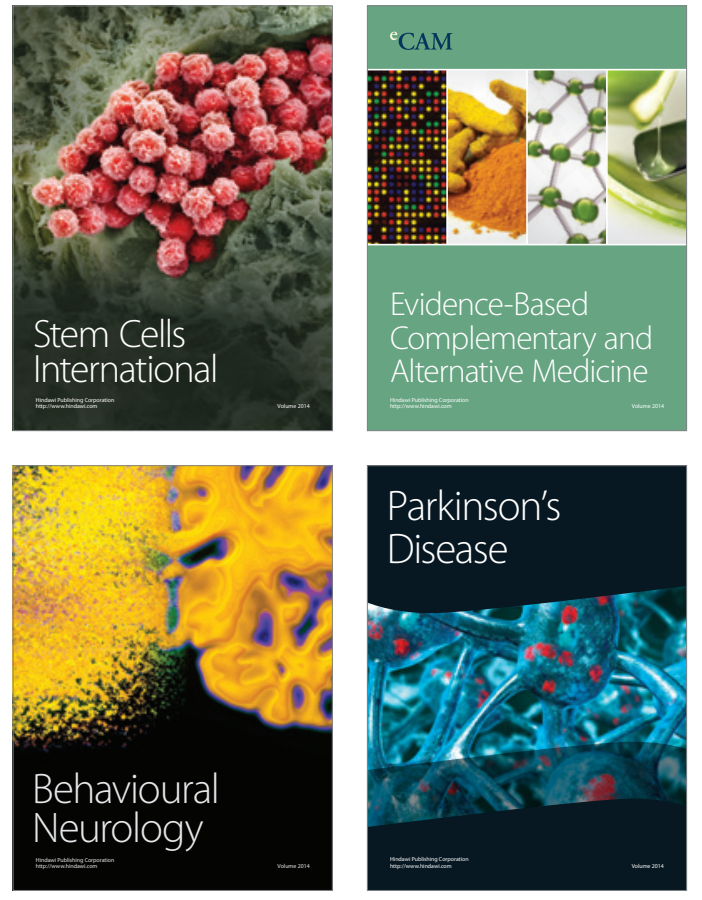
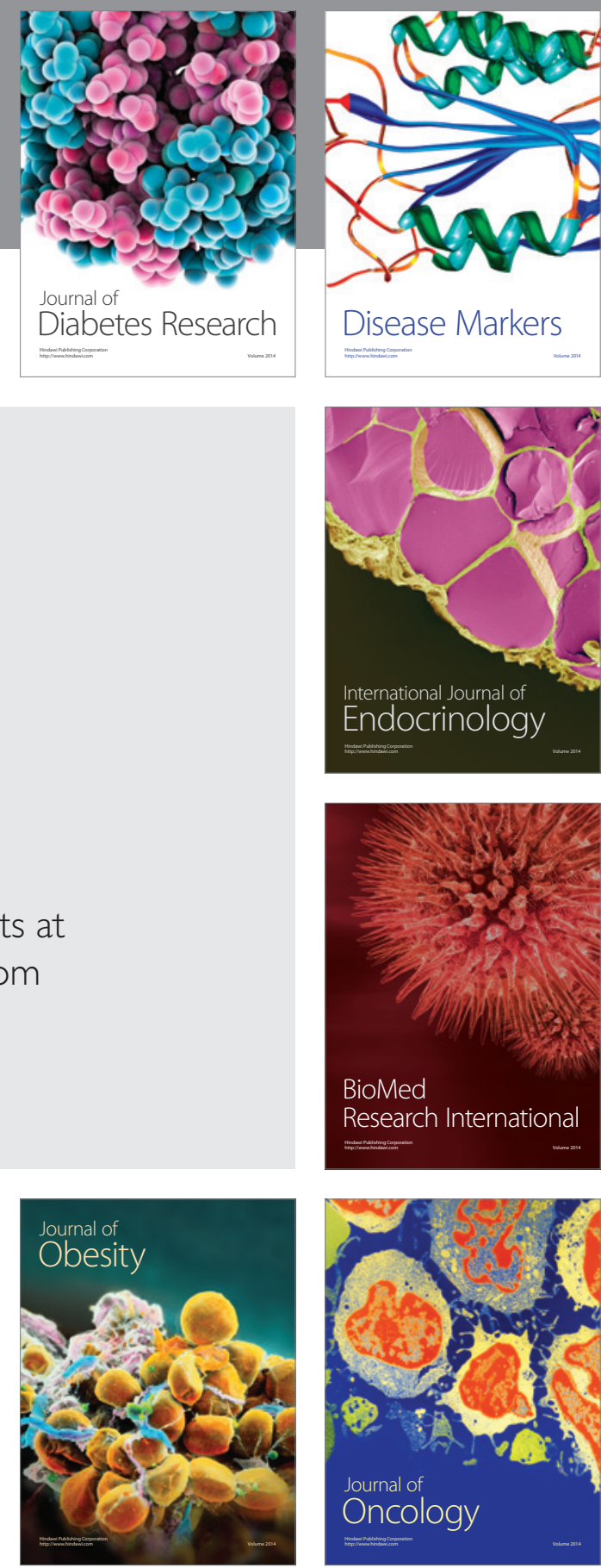

Disease Markers
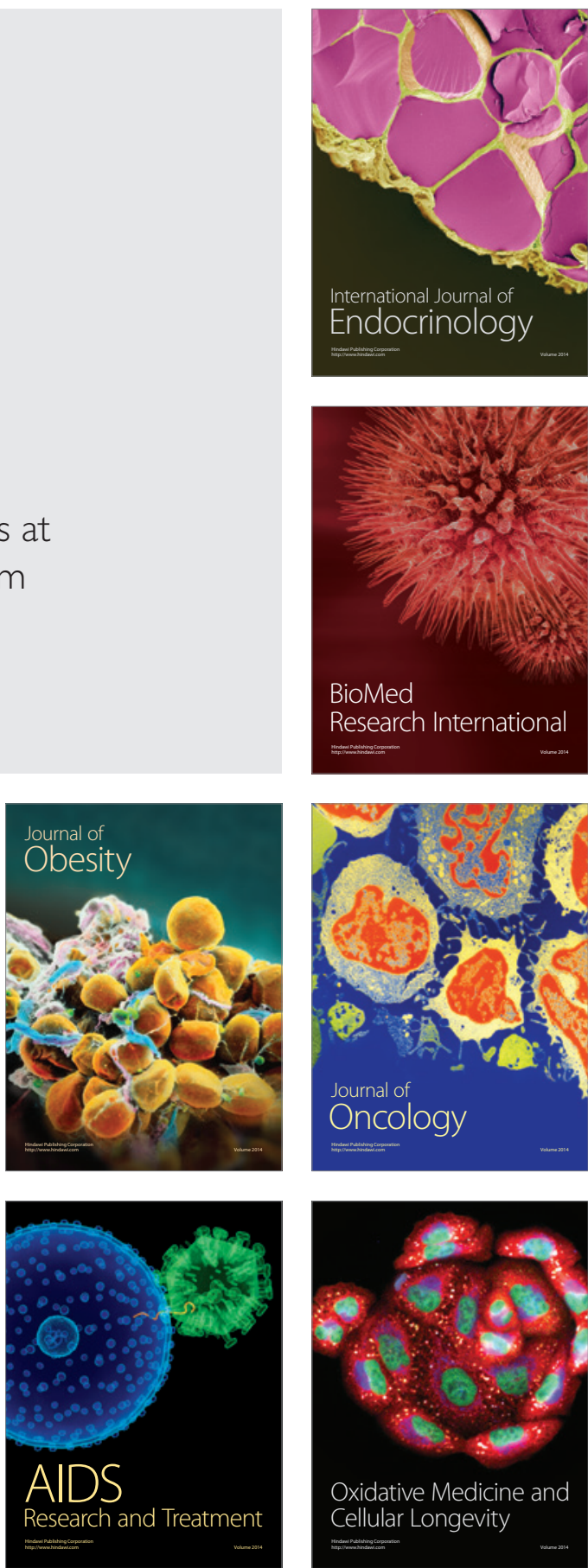